\title{
SP Picture for Scalable Video Coding
}

\author{
Jie Jia, Hae-Kwang Kim, and Hae-Chul Choi \\ Department of Computer Science, Sejong University, \\ 143-747, Seoul, Korea \\ Radio \& Broadcasting Division, ETRI, \\ 305-700, Daejeon, Korea \\ \{jiejia, hkkim\}@sejong.ac.kr, choihc@etri.re.kr
}

\begin{abstract}
This paper investigates an extension of the SP picture from the H.264/AVC to the scalable video coding (SVC), which has been recently developed and standardized as the scalable extension of the H.264/AVC. In comparison with the scalable profiles of previous video coding standards, the SVC has achieved significant improvement in both coding efficiency and scalability in temporal, spatial and fidelity, which efficiently provides coded stream wide adaptivity to dynamic network conditions as well as diverse clients. In communication environments, this efficient adaptivity can be provided by bit stream switching between different scalable layers. The current SVC supports bit stream switching only at instantaneous decoding refresh (IDR) access unit. However, in order to provide instantaneous switching capability, the IDR picture needs to be frequently coded in the SVC stream, which dramatically decreases the coding efficiency. Therefore, SP picture for the SVC is proposed in this paper for efficient bit stream switching. Performance analysis shows that the SP picture for the SVC provides an average $1.2 \mathrm{~dB}$ PSNR enhancement over the IDR picture while providing similar functionalities.
\end{abstract}

Keywords: Scalable Video Coding, bitstream switching, SP picture.

\section{Introduction}

Video applications today range from video conference, mobile video to highdefinition (HD) TV broadcast and HD DVD storage, where application environments cover mobile, wireless and wired network with various conditions as well as diverse clients and system resources. For those applications, both coding efficiency and adaptivity are the most important. To meet requirements from those applications, the scalable video coding (SVC) extension of the H.264/AVC has been developed and recently standardized by the Joint Video Team (JVT) of the ITU-T Video Coding Experts Group (VCEG) and the ISO/IEC Moving Picture Experts Group (MPEG) 1]. SVC addresses coding schemes for efficient and adaptive video storage and communication over heterogeneous network for diverse clients using available system resource. The most significant improvement of the SVC is the enhanced coding efficiency and scalability over the scalable profiles of previous video coding standards, such as MPEG-2, H.263+ and MPEG-4 part II.

D. Mery and L. Rueda (Eds.): PSIVT 2007, LNCS 4872, pp. 788 800, 2007.

(C) Springer-Verlag Berlin Heidelberg 2007 
The purpose of SVC is to provide an universally accessible bit stream which can be decoded when part of the stream is removed for adapting dynamic and various network conditions as well as user preferences. One extracted scalable bit stream constitutes another bit stream corresponding to a decreased frame rate, picture resolution or quality level. For communication applications, this adaptivity can be provided by dynamic bit stream switching between different scalable layers. The SVC provides coded stream inter-layer switching capability at instantaneous decoding refresh (IDR) access unit. However, an employment of the IDR picture decreases the coding efficiency, especially when it is frequently coded in bit stream. Referring to the H.264/AVC, a similar problem is solved by a new type picture, the SP picture which was proposed and adopted to AVC for efficient bit stream switching 23,4 .

Basically, the SP picture employs inter picture motion estimation and motion compensation in a similar way as the $\mathrm{P}$ picture. While different from the $\mathrm{P}$ picture, the SP picture allows an identical reconstruction with a pair of SP pictures. The two SP pictures, denoted as the primary SP picture (the SP picture for non-switching) and the secondary SP picture (the SP picture for switching), are predicted from reference picture decoded from the current stream and the stream switching from, respectively [4 5] 6]. SP pictures achieve this identical reconstruction by lossless coding a secondary SP picture with a quantized primary SP picture as input. Note, only a primary SP picture is coded and transmitted in a bit stream. A secondary SP picture is only transmitted and decoded when switching occurs, at this time, no primary SP picture is decoded. Intensive simulation results show that the SP picture outperforms the IDR picture on coding efficiency regarding the functionality of bit stream switching [7. Therefore, in this paper, we extend the SP picture from AVC to SVC for an efficient SVC bit stream switching capability.

This paper is structured as follows. Sec. 2 presents an overview of the SVC scalable coding structure as well as a summary of the SP picture for AVC. Sec. 3 investigates the SP picture for SVC. Moreover, a proof of the drift-free switching via SP picture for SVC is also given. Sec. 4 analyzes the performance of SP picture for SVC, followed by a conclusion drawn in Sec. 5 .

\section{SVC Overview and SP Picture Basics}

SVC was designed as an scalable extension of the H.264/AVC. The SVC exploits most of the AVC techniques and further develops inter-layer prediction which greatly contributes to the enhanced coding efficiency. SVC provides scalability in temporal, spatial as well as fidelity. A hierarchical prediction structure enables the SVC a flexible temporal scalability. A layered coding structure with the enhanced inter-layer prediction contributes to the spatial and fidelity scalability. In the following of this section, a brief overview of the SVC is given, followed by a concept explanation of SP picture. For detailed description of SVC, the reader is referred to the draft standard [1] and an overview of the SVC [8]. 


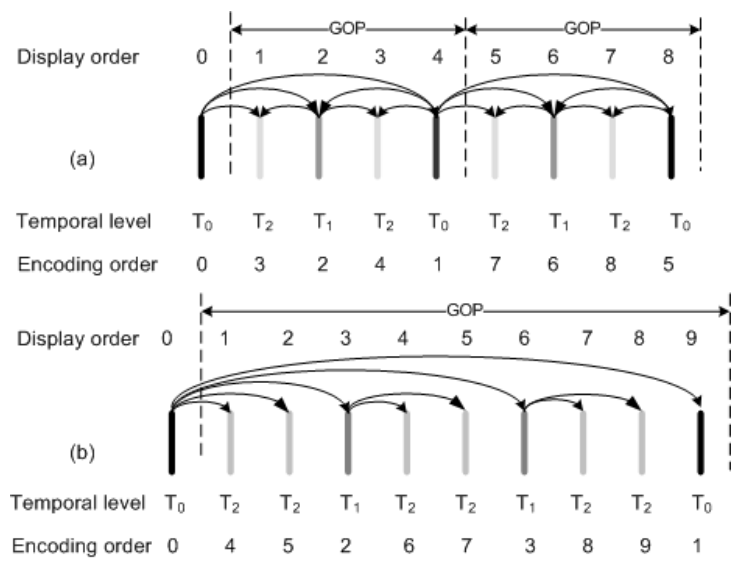

Fig. 1. Illustration of hierarchical prediction structure. (a) dyadic temporal scalability with hierarchical B picture, (b) non-dyadic scalability with hierarchical $\mathrm{P}$ picture.

\subsection{Temporal Scalability}

SVC provides both dyadic and non-dyadic temporal scalability by hierarchical $\mathrm{B} / \mathrm{P}$ picture 9]. Different temporal layers are identified by temporal level identification, which starts from 0, representing temporal base layer, and is increased by 1 for every enhancement on temporal layer. Fig. 1 illustrates the hierarchical prediction structure for enabling temporal scalability. Fig. 11(a) presents a dyadic coding structure with hierarchical B picture which provides another two independently decodable sub-sequences with $1 / 4$ and $1 / 2$ of the full temporal resolution. Fig. 1(b) describes a non-dyadic prediction structure based on hierarchical $\mathrm{P}$ picture where another two sub-sequences can be independently decoded with $1 / 9$ and $1 / 3$ of the full temporal resolution.

A principle for this hierarchical prediction is that a base temporal layer pictures can only be predicted from previous decoded picture(s) with the same temporal level, and an enhancement temporal layer picture with temporal level $T_{i}$ can be predicted from picture(s) with temporal level $T_{k}$, where $\mathrm{k} \leq \mathrm{i}$. Note, the hierarchical prediction structure can also be combined with the multiple reference picture concept from the H.264/AVC. Besides the flexible temporal scalability, the hierarchical prediction structure is also possible to provides a zero structure delay which is supported by hierarchical $\mathrm{P}$ picture, as illustrated in Fig. 1 (b).

\subsection{Spatial Scalability}

In order to support spatial scalability, the SVC employs a multi-layer coding structure, which is also used in MPEG-2, H.263 and MPEG-4 Visual. But different from previous standards, the SVC encodes pictures of spatial enhancement layer with both intra-layer motion compensated prediction and layer-specific 
inter-layer prediction. For improving SVC coding efficiency, an enhanced interlayer prediction has been developed which consists of inter-layer motion prediction, inter-layer residual prediction and inter-layer intra prediction [10.

In order to employ base layer information as much as possible for the spatial enhancement layer coding, the SVC includes a new macroblock (MB) type, denoted as BlSkip. With this type for enhancement layer MB, both MB partition and motion information including reference frame and motion vector are derived from collocated base layer MB. This is the inter-layer motion prediction.

Besides the inter-layer motion prediction, an MB based residual signal prediction is also provided. When this inter-layer prediction is employed, the block-wise up-sampled base layer residual signal where a bi-linear filter is applied, is used as the prediction signal for the enhancement layer residual signal coding.

Regarding the BlSkip mode, when the corresponding block in base layer is fully located within an intra-coded MB, the current enhancement layer MB is coded with an inter-layer intra prediction. The prediction signal for the enhancement layer $\mathrm{MB}$ is obtained by up-sampling the reconstructed intra-coded block in base layer. Note, constrained intra prediction is always applied to layers that are employed for inter-layer prediction, so that only a single motion compensation loop is needed for decoding.

\subsection{Fidelity Scalability}

SVC provides fidelity scalability in the form of coarse gain scalability (CGS) and medium gain scalability (MGS) [11. The CGS employs the same intra-layer prediction and a similar inter-layer prediction as that used for spatial scalable coding without up-sampling. The number of supported quality levels is identical to the number of layers. MGS employs a similar motion compensated prediction structure as that for FGS 12, but without bit-plane coding. Therefore, switching between different quality levels is virtually possible in any access unit for MGS.

\subsection{SP Picture Basics}

SP picture was firstly proposed by Karczewicz and Kurceren in [7] for AVC bit stream switching, error resilience etc. A pair of SP picture provide an identical reconstruction even their motion compensated predictions are performed from different reference picture.

When a primary SP picture is coded, the constructed signal is requantized with a finer quantizer than that used for the residual signal quantization. Then the requantized signal is reconstructed and sent to decoded picture buffer, where pictures are further used as the reference for the following picture prediction. Therefore, in general, the primary SP picture is slightly less efficient in compression than the regular $\mathrm{P}$ picture, but significantly more efficient than the IDR picture thanks to the motion compensation.

A constructed primary SP picture prior to deblocking process is fed as an original signal to a secondary SP picture encoder. Similar to that for primary $\mathrm{SP}$ picture coding, the motion compensation for secondary SP picture is also 
performed in transform domain. After that, the residual signal of secondary SP picture is lossless coded, so that a mismatch free construction for the primary SP picture can be obtained by the secondary SP picture. Usually, the secondary $\mathrm{SP}$ picture is much less efficient than the regular $\mathrm{P}$ picture. For further description about the SP picture, readers are referred to 4$][5]$.

\section{SP Picture for SVC}

In this section, we first discuss applications of SP picture for SVC, then present a detailed description of encoding process for inter-picture coded MBs in enhancement SP picture. Finally, an analytical proof of the drift-free switching is given.

\subsection{Applications}

SP picture for AVC provides efficient drift-free switching between different streams. This provides SP picture wide applications such as error recovery, fast forward/ backward as well as bit stream switching. An extension of SP picture from AVC to SVC not only further exploits features of SP picture, but also, more importantly, provides a more efficient and flexible inter-layer switching for SVC.

Considering real-time video communication, say video conference, compressed video streams are transmitted under various network conditions which can be wireless or wired heterogeneous network. For those application, the available bandwidth is always varying due to the dynamic network condition. SVC provides multiple decodable bit stream which can be decoded into video sequences with different frame rate, spatial resolution and/or quality level which is controlled by scalable layer. This feature significantly enhances the adaptivity of scalable coded streams to different network conditions as well as diverse clients.

However, when real-time is concerned, the dynamic network requires coded stream to timely adapt to variations of the bandwidth available to client. For scalable coded streams, this can be obtained by inter-layer switching, but instantaneous is needed. IDR picture is one of the solutions. But, unfortunately, as described before, a frequent usage of IDR picture significantly decreases the coding efficiency. With SP picture for SVC, thanks to its significantly enhanced coding efficiency in comparison with the IDR picture, a frequent employment of SP picture is possible with just slightly performance decrease. Usually, primary SP picture is transmitted and decoded. When switching occures, a secondary SP picture is transmitted and decoded which provides an exact same reconstruction as that of the primary SP picture. This enables scalable coded stream a timely adaptivity to the dynamic network conditions. Regarding the performance mentioned above, it can be further referred to Sec. 4 .

\subsection{Encoding Process for SP Picture for SVC}

SP picture for SVC was first proposed and presented in [13. The most significant difference between the SP picture for AVC and the SP picture for SVC lies in 


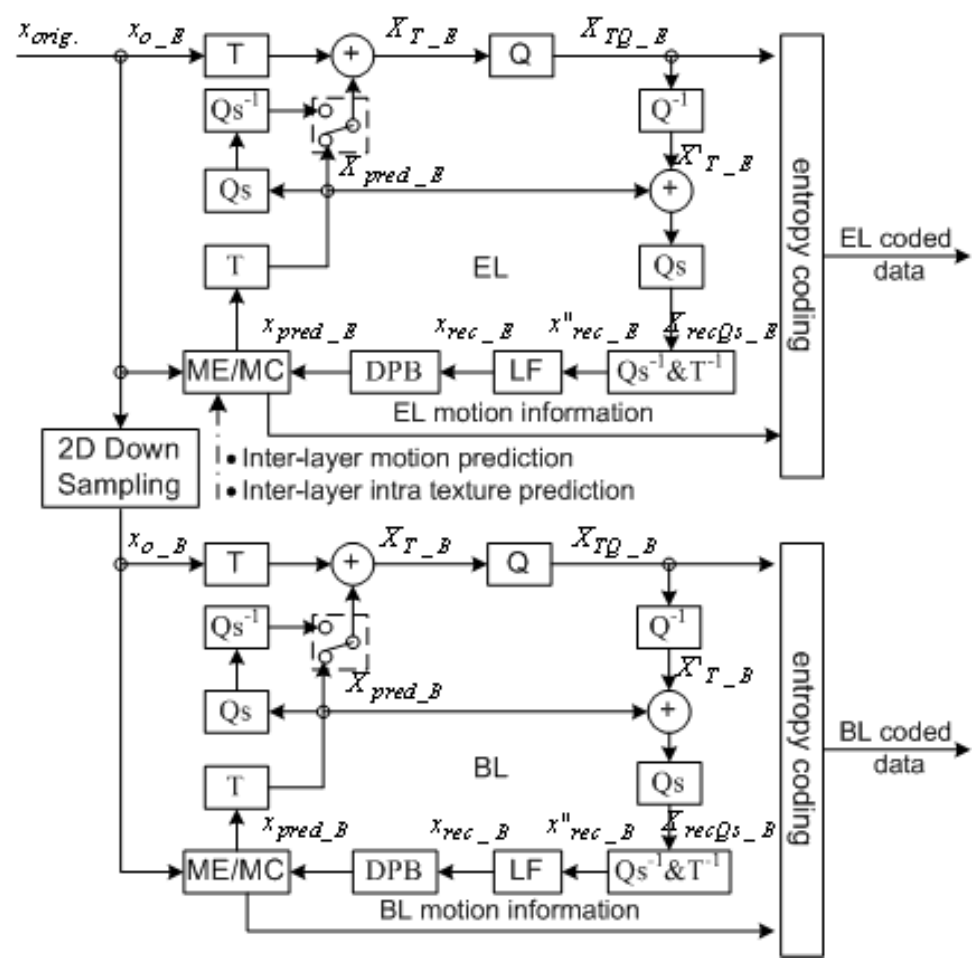

Fig. 2. SVC primary SP picture coding structure

the inter-layer prediction for enhancement SP picture coding. Fig. 2 and Fig. 3 illustrates a schematic block diagram of the encoding process for primary SP picture and secondary SP picture, respectively. In both of the figures, two spatial layers are assumed to be scalably coded. Note, the SVC is AVC compatible at base layer. Therefore, the SP picture for SVC at base layer is same as the regular SP picture for AVC.

In Fig. 2 and Fig. 3. T, $T^{-1}, \mathrm{Q}$, and $Q^{-1}$ represents the transform, inverse transform, quantization and de-quantization process, respectively. $\mathrm{ME} / \mathrm{MC}$ indicates motion estimation and motion compensation. Loop filter (LF) refers to deblocking filter. Decoded picture buffer (DPB) holds encoder reconstructed pictures which are used as reference for coding $\mathrm{P}$ and $\mathrm{B}$ pictures later.

As shown in Fig. 2, when primary SP picture is coded, basically, the downsampled original signal is firstly encoded in base layer and later the original signal is coded in enhancement layer. For each layer, two quantizers are employed. The coarser one is used for residual signal quantization, while the finer one is used for motion-compensated prediction and construction signal quantization, which is also used for the secondary SP picture coding, see Fig. 3. The finer quantizer is designed for reducing the secondary SP picture stream size with the cost of a decreased primary picture quality. 


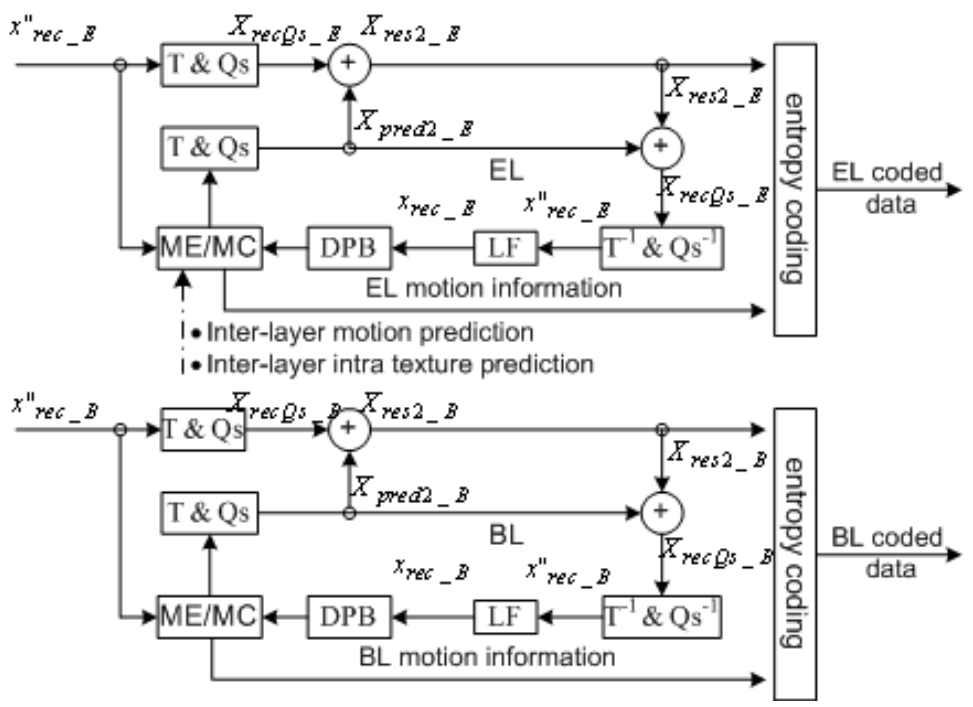

Fig. 3. SVC secondary SP picture coding structure

Regarding the enhancement SP picture coding process, firstly, ME/MC is performed, then a transform is applied separately to the prediction signal and original signal. After that, residual signal is obtained in the transform domain. Note, an inter-layer prediction is employed in the $\mathrm{ME} / \mathrm{MC}$ process for the enhancement SP picture coding. Similar to the inter-layer prediction which is used for enhancement $\mathrm{P}$ picture coding, both inter-layer motion prediction and interlayer intra prediction are utilized. But different from enhancement $\mathrm{P}$ picture, enhancement SP picture does not include inter-layer residual prediction. A description of inter-layer prediction was given in Sec. 2.2

Primary SP picture is designed to efficiently encode a picture that can have an identical reconstruction even that is predicted from different reference picture. Secondary SP picture is designed to encode a picture, the reconstruction of which is identical to that of primary SP picture. For this purpose, the construction signal in primary SP picture coding process prior to LF is fed as the input to secondary SP picture coding process, as shown in Fig. 3. Note, similar to the enhancement primary SP picture coding, only inter-layer motion prediction and inter-layer intra prediction are employed in the enhancement layer $\mathrm{ME} / \mathrm{MC}$ process. A further explanation on the drift-free switching is provided in Sec. 3.3 .

\subsection{Drift-Free Switching by SP Picture for SVC}

This section gives an analytical proof to the drift-free switching for SVC provided by the proposed SP picture. As the base layer of SVC is AVC compatible, in the following analysis, a proof of the identical reconstruction provided by enhancement SP pictures is given. 
As discussed before, in order to obtain a mismatch free reconstruction between the primary SP picture and the secondary SP picture, the constructed signal $x{ }^{\prime} r_{\text {rec }}$ of primary SP picture prior to deblocking process, as shown in Fig. 2, is used as the input to the secondary SP picture coding process.

Referring to the coding process of primary SP picture, $x{ }^{\prime}{ }_{r e c} E$ can be expressed as (1).

$$
x_{\text {rec_E }}^{\prime \prime}=T^{-1} Q_{s}^{-1}\left\{Q_{s}\left[X_{T_{-} E}^{\prime}+X_{\text {pred_E }}\right]\right\}
$$

Where $X_{T_{-} E}^{\prime}$ and $X_{\text {pred_E }}$ is the de-quantized residual signal in transform domain and the transformed prediction signal, respectively. Moreover, $T^{-1}$ and $Q_{s}^{-1}$ represent the inverse transform and de-quantizer.

In the secondary SP picture coding process, the input signal $x^{\prime \prime} r e c_{-} E$ is transformed and quantized with quantizer $Q_{s}$. Combining (1), this process can be expressed as (2).

$$
\begin{aligned}
Q_{s} \cdot T\left(x_{r e c_{-} E}^{\prime \prime}\right)= & Q_{s} \cdot T\left(T^{-1} Q_{s}^{-1}\left\{Q_{s}\left[X_{T \_E}^{\prime}+X_{\text {pred_E }}\right]\right\}\right) \\
& =Q_{s} \cdot Q_{s}^{-1}\left\{Q_{s}\left[X_{T_{-} E}^{\prime}+X_{\text {pred_E }}\right]\right\} \\
& =Q_{s}\left[X_{T \_E}^{\prime}+X_{\text {pred_E }}\right]
\end{aligned}
$$

Followed the transform and quantization, a transform domain motion compensation is performed, which generates the corresponding residual signal that will be lossless coded for the secondary SP picture. Thanks to the lossless coding process, a construction signal in transform domain can be obtained which is exactly same as the quantized input signal in transform domain. Then, after the de-quantization and inverse transform, a construction signal prior to deblocking process can be obtained. This process combined with (2) is given by (3)

$$
\begin{aligned}
T^{-1} Q_{s}^{-1}\left\{X_{r e c Q s_{-} E}\right\}= & T^{-1} Q_{s}^{-1}\left\{Q_{s}\left[X_{T_{-} E}^{\prime}+X_{\text {pred_E }}\right]-X_{\text {pred } 2_{-} E}+X_{\text {pred } 2_{-} E}\right\} \\
& =T^{-1} Q_{s}^{-1}\left\{Q_{s}\left[X_{T_{-} E}^{\prime}+X_{\text {pred_E }}\right]\right\}
\end{aligned}
$$

It can be seen that (3) and (11) represent the exact same signal. Therefore an exact same reconstruction can be obtained by decoding the primary SP picture and the secondary SP picture. This guarantees a drift-free switching between different scalable coded streams.

\section{Performance Analysis}

To illustrate the coding efficiency performance of the SP picture for SVC, a software implementation of the proposed SP picture was done based on the SVC software JSVM 8 [14. The implemented software was submitted to JVT with 15. Simulations under the SVC coding efficiency test condition [16] were performed. All eight of the standard test sequences are tested. In the simulation, CABAC is chosen as the entropy coding method, and rate-distortion optimization is employed.

Three test sets are performed in this paper. Those consist of the performance comparison between SP picture and Intra picture, the comparison between SP 


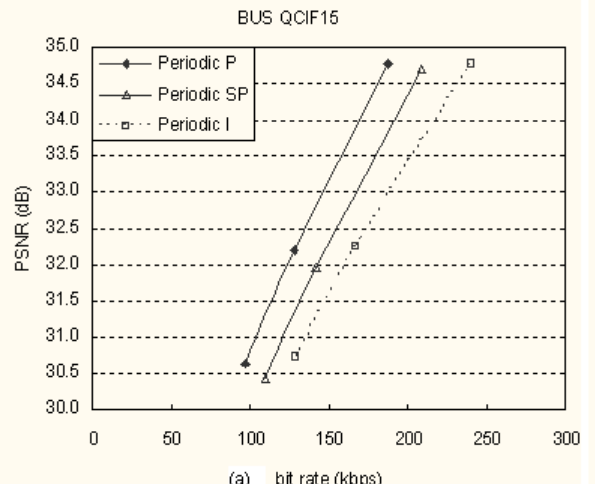

(a) bit rate (kbps)

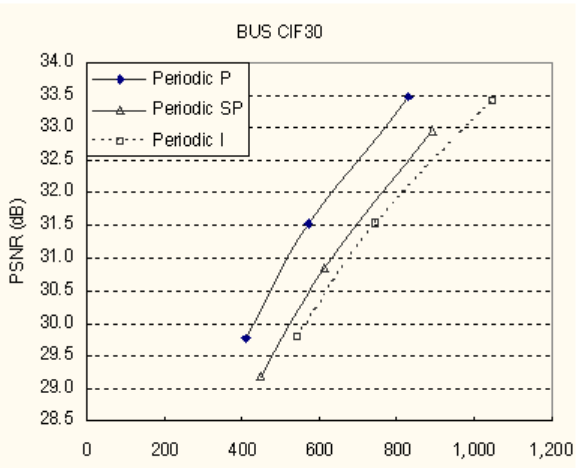

(b) bit rate (kbps)

Fig. 4. Performance comparison for Bus sequence with GOP size $=8$, key picture is encoded as Inter P, SP and Intra picture, respectively. $Q P_{s p}=Q P_{p}-2, Q s=Q P_{p}-5$ (a) base layer, (b) enhancement layer.

picture and EIDR picture, and the comparison between SP pictures which are coded with different $Q P_{s p}$ and $Q_{s}$ values. Fig. 4 to Fig. 7 and Tab. 1 to Tab. 2 illustrate the simulation results.

Basically, the simulation results report that the proposed SP picture coding scheme enhances the coding efficiency performance of SVC. Fig. 4 and Fig. 5 compare the coding efficiency performance between the inter P, SP and Intra pictures. For all three cases, pictures with the lowest temporal level are coded as Inter P, SP and Intra pictures, respectively, which are denoted with periodic P, periodic SP and periodic I. Similar notations are also applied to the following figures in this section. Regarding the "Bus" sequence, an average $0.8 \mathrm{~dB} \mathrm{im}$ provement can be observed from the simulation results by the SP picture over the Intra picture for the base layer. Furthermore, a 0.4 dB PSNR enhancement can be observed at the enhancement layer. Simulation results for the "Mobile" sequence report an average $1.7 \mathrm{~dB}$ and $1.2 \mathrm{~dB}$ PSNR improvement of the SP picture over the Intra picture for the base layer and the enhancement layer respectively.

Fig. 6illustrates the performance comparison between the SP picture and the EIDR picture. In the simulation, both of the SP picture and the EIDR picture are periodically coded for the enhancement layer. The GOP size of 8 is employed. From the simulation results, it can be observed that the SP picture improves the coding efficiency performance by an average $1.6 \mathrm{~dB}$ PSNR enhancement over the EIDR picture. Note, as the SP picture and the EIDR picture are only encoded for the enhancement layer, the performance regarding the base layer is same for all three of them.

Fig. 7 compares the SP picture performance when different quantization parameters are employed. Basically, two sets of QP values are tested in the simulation. The curve with a triangle mark presents the performance of SP picture which is 

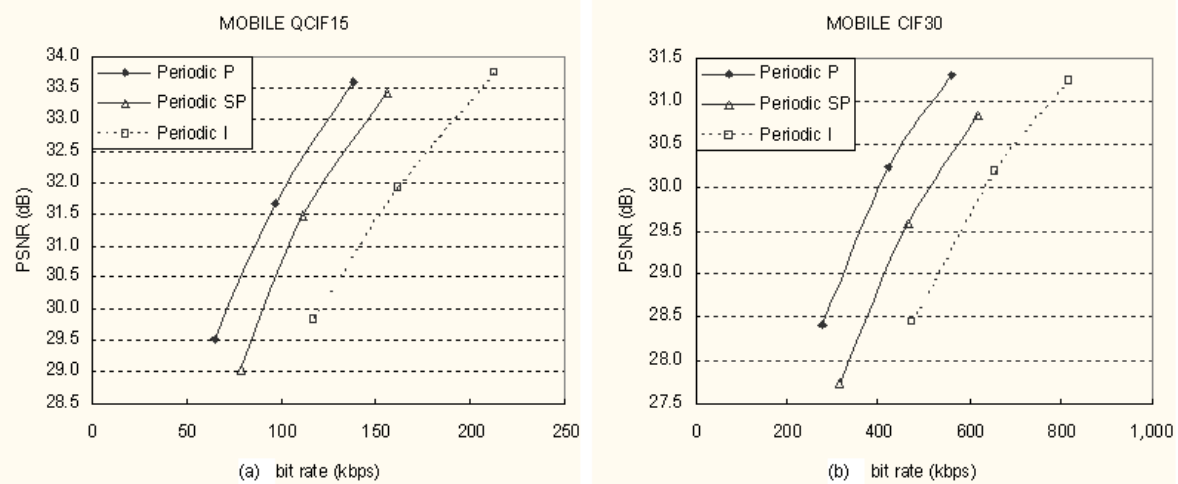

Fig. 5. Performance comparison for Mobile sequence with GOP size $=16$, key picture is encoded as Inter $\mathrm{P}, \mathrm{SP}$ and Intra picture, respectively. $Q P_{s p}=Q P_{p}-2, Q s=Q P_{p}-5$ (a) base layer, (b) enhancement layer.

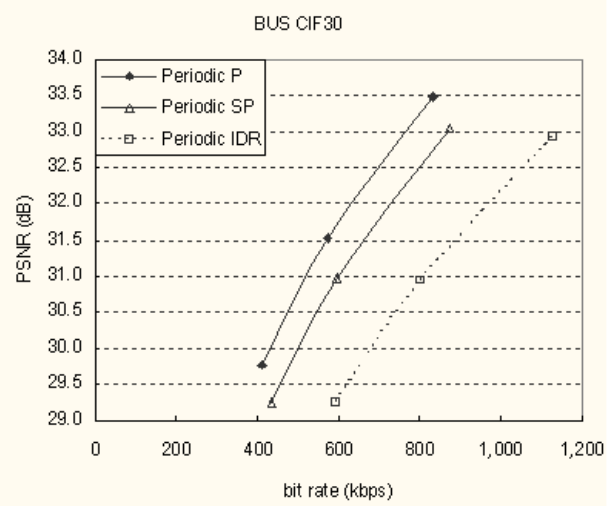

Fig. 6. Performance comparison between SP picture and EIDR picture, Bus sequence, GOP size $=8, Q P_{s p}=Q P_{p}-2, Q s=Q P_{p}-5$

coded with $Q P_{s p}=Q P_{p}-2$ and $Q s=Q P_{p}-5$. The curve with a cross mark presents the performance of SP picture which is coded with $Q P_{s p}=Q P_{p}-1$ and $Q s=Q P_{p}-10$. From the figure, it can be seen that lower Qs improves the performance of the primary SP picture. However, it increases the bits used for representing the secondary $\mathrm{SP}$ picture. Therefore, in the previous simulations, the quantization parameters for the $\mathrm{SP}$ picture are set as $Q P_{s p}=Q P_{p}-2$ and $Q s=Q P_{p}-5$.

Tab. 1 and Tab. 2 illustrate comparisons on average coded picture size between the IDR picture and the secondary SP picture for the "Bus" and "Mobile" 

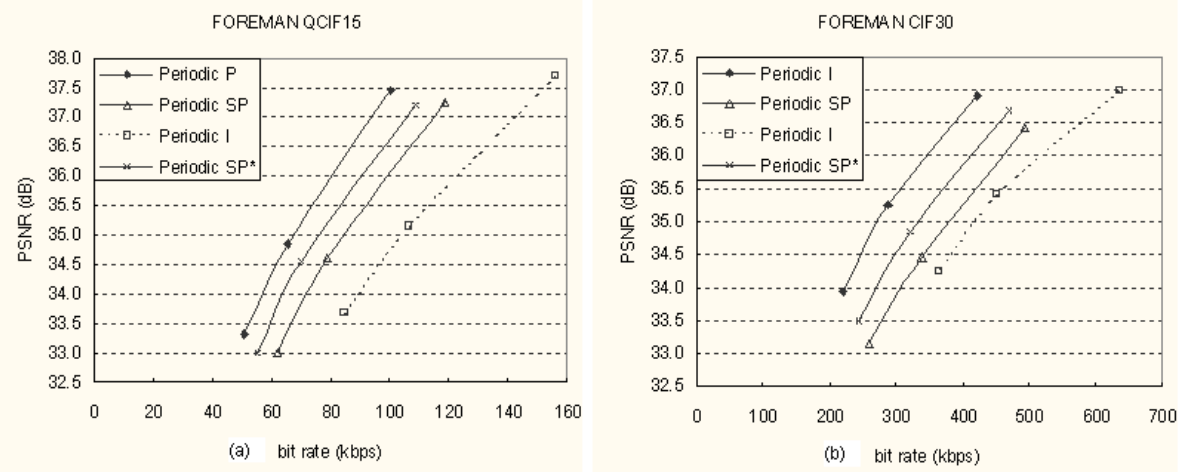

Fig. 7. Performance comparison between SP pictures which are coded with different $Q P_{s p}$ and $Q s$. Foreman sequence, GOP size $=8$, (a) base layer, (b) enhancement layer.

Table 1. Comparison of average coded I and SP picture size for Bus sequence

\begin{tabular}{|c|c|c|c|c|c|c|}
\hline Bus & & 30 & & 33 & & 35 \\
\hline \multirow[t]{2}{*}{$\overline{\mathrm{IDR}}$} & & 33162 & & 24536 & & 19485 \\
\hline & 27 & 34198 & 30 & 26110 & 32 & 21266 \\
\hline \multirow[t]{2}{*}{ SP } & 33 & 32792 & 36 & 23604 & 38 & 18590 \\
\hline & 36 & 34485 & 39 & 24917 & 41 & 19656 \\
\hline
\end{tabular}

Table 2. Comparison of average coded I and SP picture size for Mobile sequence

\begin{tabular}{ccccccc}
\hline Mobile & & 31 & & 33 & & 37 \\
\hline IDR & & 50786 & & 41438 & & 28450 \\
& 28 & 34280 & 30 & 27338 & 34 & 17133 \\
SP & 34 & 33311 & 36 & 26274 & 40 & 16468 \\
& 37 & 37988 & 39 & 32051 & 43 & 22541 \\
\hline
\end{tabular}

sequences. In this simulation, sequences are coded at QCIF format. A bit stream switching between different quality layers are employed. The QP values given in the first row within each table are the QP values used for the target bit stream coding. The $\mathrm{QP}$ values listed in each column are the $\mathrm{QP}$ values used for coding the stream from which the target bit stream is switched. From the tables, it can be seen that a similar or less coded picture size can be generally obtained for the "Bus" sequence with the secondary SP picture in comparison to the IDR picture. While regarding the "Mobile" sequence, only around $2 / 3$ of the coded IDR picture size is needed for representing the secondary SP picture. This is due to the reason that the number of bits used for representing a picture depends on the picture content. A complex content picture, such as the "Mobile" sequence, 
requires more bits to represent the pictures than a simple content picture does. This is more obvious between the Intra picture coding and the Inter picture coding, say the IDR picture and the SP picture.

\section{Conclusion}

This paper presents application, design, proof of drift-free switching and performance analysis of SP picture for SVC. An overview of SVC and SP picture is also given. The proposed SP picture for SVC further exploits adaptivity of SVC to various dynamic network conditions as well as diverse clients. The SP picture for SVC is based on the SP picture for AVC. Different from both the regular $\mathrm{SP}$ picture and the $\mathrm{P}$ picture, the $\mathrm{SP}$ picture for $\mathrm{SVC}$ employs not only intra-layer inter prediction, intra prediction, but also inter-layer intra prediction and inter-layer motion prediction. Those contributes to the final enhanced coding efficiency performance in comparison with the IDR picture. Simulation results show that an average $1.2 \mathrm{~dB}$ PSNR enhancement can be observed by the $\mathrm{SP}$ picture for SVC over the IDR picture.

Acknowledgments. This work was supported by the Korea Science and Engineering Foundation (KOSEF) grant funded by the Korea government (MOST)(No. R01-2007-000-11078-0) and ETRI (Electronics and Telecommunications Research Institute).

\section{References}

1. Wiegand, T., Sullivan, G., Reichel, J., Schwarz, H., Wien, M.: Joint draft 10 of SVC amendment. In: Joint Video Team Meeting, San Jose, CA, USA, Doc. JVTW201 (2007)

2. ISO/IEC JTC 1: Advanced video coding for generic audio-visual services, ITU-T Recommendation H.264 and ISO/IEC 14496-10 (MPEG4-AVC), Version 4 (2005)

3. Wiegand, T., Sullivan, G.J., Bjøntegaard, G., Luthra, A.: Overview of the H.264/AVC video coding standard. IEEE Trans. on Circuits and Systems for Video Technology 13(7), 560-576 (2003)

4. Karczewics, M., Kurceren, R.: The SP- and SI- frames design for H.264/AVC. IEEE Trans. on Circuits and Systems for Video Technology 13(7), 637-644 (2003)

5. Setton, E., Girod, B.: Rate-distortion analysis and streaming of SP and SI frames. IEEE Trans. on Circuits and Systems for Video Technology 16(6), 733-743 (2006)

6. Sun, X., Wu, F., Li, S., Shen, G., Gao, W.: Drift-free switching of compressed video bitstreams at predictive frames. IEEE Trans. on Circuits and Systems for Video Technology 16(5), 565-576 (2006)

7. Karczewics, M., Kurceren, R.: A proposal for SP-frames. In: Video Coding Expert Group Meeting, Eibsee, Germany, Doc. VCEG-L27 (2001)

8. Schwarz, H., Marpe, D., Wiegand, T.: Overview of the scalable video coding extension of the H.264/AVC standard. In: Joint Video Team Meeting, San Jose, CA, USA, Doc. JVT-W132 (2007) 
9. Schwarz, H., Marpe, D., Wiegand, T.: Hierarchical B pictures. In: Joint Video Team Meeting, Poznan, Poland, Doc. JVT-P014 (2005)

10. Schwarz, H., Marpe, D.H., Wiegand, T.: SVC core experiment 2.1: Inter-layer prediction of motion and residual data. In: Moving Picture Experts Group Meeting, Redmond, WA, USA, ISO/IEC JTC 1/SC 29/WG 11, Doc. M11043 (2004)

11. Kirchhoffer, H., Schwarz, H., Wiegand, T.: SVC core experiment 1: Simplified FGS. In: Joint Video Team Meeting, San Jose, CA, USA, Doc. JVT-W090 (2007)

12. Winken, M., Schwarz, H., Marpe, D., Wiegand, T.: Adaptive refinement of motion information for fine-granular SNR scalable video coding. In: European Symposium on Mobile Media Delivery, EuMob 2006, Alghero, Italy, September 2006 (2006)

13. Jia, J., Kim, H.K., Choi, H.C., Kim, J.-G.: SP picture for SVC switching. In: Joint Video Team Meeting, Marrakech, Morocco, Doc. JVT-V045 (2007)

14. Vieron, J., Wien, M., Schwarz, H.: JSVM8 software. In: Moving Picture Experts Group Meeting, Hangzhou, China, ISO/IEC JTC 1/SC 29/WG 11, Doc. N8457 (2006)

15. Jia, J., Kim, H.K., Choi, H.C., Kim, J.-G.: SVC core experiment 2: Switching for SVC. In: Joint Video Team Meeting, Hangzhou, China, Doc. JVT-U302 (2006)

16. Wien, M., Schwarz, H.: Test conditions for SVC coding efficiency and JSVM performance evaluation. In: Joint Video Team Meeting, Nice, France, Doc. JVT-Q205 (2005) 